\title{
The role of cytoplasmic p57 in invasion of hepatocellular carcinoma
}

\author{
Hui Guo ${ }^{\dagger}$, Yi Li ${ }^{\dagger}$, Tao Tian, Lili Han, Zhiping Ruan, Xuan Liang, Wenjuan Wang and Kejun Nan
}

\begin{abstract}
Background: Our previous research suggested that p57 downregulation could accelerate the growth and invasion of hepatocellular carcinoma in vitro and in vivo.

Aim: To evaluate the role of cytoplasmic p57 and its regulatory mechanism during hepatocellular carcinoma invasion.

Methods: We examined the subcellular localization of p57 by immunohistochemistry in 45 pairs of cancerous tissues and adjacent non-cancerous tissues. Moreover, we generated stable p57 knockdown hepatoma cell lines to investigate the mechanism of cytoplasmic p57-mediated regulation of invasion by immunoprecipitation, confocal immunofluorescence microscopy and western blot of nuclear and cytoplasmic extracts.

Results: Our results showed that cytoplasmic expression of p57 was reduced in specimens from patients with capsular invasion and metastasis $(P<0.05)$. Moreover, the level of $p$-cofilin was decreased in the group lacking cytoplasmic $p 57$ expression $(P<0.05)$. Co-expression of p57 and p-cofilin was reduced in specimens from patients with tumors at later stages $(I I+\mid \mathrm{V})$, tumors showing capsular invasion and metastatic tumors. We further observed that p57 downregulation decreased the assembly of p57 and LIM domain kinase 1 and its kinase activity, subsequently reducing the level of p-cofilin in the cytoplasm.
\end{abstract}

Conclusions: Cytoplasmic p57 might be a key regulator in hepatocellular carcinoma invasion via the LIM domain kinase $1 / p$-cofilin pathway.

Key words: Cytoplasmic p57, Invasion, Hepatocellular carcinoma

\section{Background}

p57 is a cyclin-dependent kinase inhibitor (CKI) that belongs to the Cip/Kip family, which includes p21, p27 and p57. The human $p 57$ gene is located on chromosome 11p15.5, a region implicated in Beckwith-Wiedemann syndrome, a familial cancer syndrome [1]. Low expression of p57 protein has been observed in human tumors, including carcinomas of the prostate, bladder, gastrointestinal tract, pancreas and breast, which suggests that p57 is an important tumor suppressor [2-4]. An increasing number of studies have shown that p57 is a multifunctional protein that is also involved in the regulation of transcription, apoptosis, differentiation and motility $[5,6]$.

\footnotetext{
* Correspondence: nankj@163.com

${ }^{\dagger}$ Equal contributors

Department of Oncology, The First Affiliated Hospital, College of Medicine of

Xi'an Jiaotong University, No. 277 Yanta West Road, Yanta District, Xi'an,

Shaanxi Province 710061, P.R. China
}

(c) 2015 Guo et al. Open Access This article is distributed under the terms of the Creative Commons Attribution 4.0

Whereas the function of Cip/Kip proteins as inhibitors of the cell cycle is well characterized in the nucleus, these proteins also seem to regulate cytoskeletal function in the cytoplasm. The Cip/Kip proteins have been reported to modulate the RhoA/ROCK/LIMK/cofilin signaling pathways involved in tumor invasion and metastasis. Surprisingly, p21 and p27 have been shown to interact with Ras homolog gene family member A (RhoA) and rho-associated, coiled-coil-containing protein kinase 1 (ROCK1) in the cytoplasm and to accelerate cancer cell invasion and metastasis, suggesting a role for these proteins that opposes the typical role of tumor suppressors in the nucleus [7]. Although several studies have reported that $\mathrm{p} 57$ can interact with LIM domain kinase (LIMK1) to affect actin cytoskeleton dynamics, the role of cytoplasmic p57 in the regulation of tumor cell invasion is unclear [8]. 
Hepatocellular carcinoma (HCC) is the sixth most prevalent cancer in the world and the third leading cause of cancer-related mortality [9]. The main reason for the poor prognosis observed in HCC patients is the highly proliferative and metastatic activities of $\mathrm{HCC}$ cells, which are the result of the deregulation of multiple signaling pathways [10]. Our previous research suggests that the downregulation of p57 accelerates the growth and invasion of HCC, indicating that p57 is an important tumor suppressor in HCC development [11]. Moreover, cytoplasmic expression of p57 was observed in HCC tissue and HCC cell lines. We are thus interested in further investigating the role and mechanism of cytoplasmic p57 in the invasion of HCC.

\section{Methods}

\section{Patients and samples}

A total of 45 paired HCC specimens and adjacent noncancerous specimens were obtained from surgical resections performed at the First Affiliated Hospital, College of Medicine, Xi'an Jiaotong University. The study participants included 36 males and 9 females with a mean age of $48.33 \pm 10.55$ years (range, 29-77 years). The other clinicopathologic data are presented in Table 1. The pathological type of each specimen was confirmed by independent pathologists. None of the patients received radiotherapy or chemotherapy prior to surgery. The TNM stage was determined using the 2002 Union International Centre Cancer (UICC) criteria. Tumor cellular differentiation was identified by Edmondson's classification. The study was approved by the Conduct of
Human Ethics Committee of the First Affiliated Hospital, College of Medicine, Xi'an Jiaotong University. Informed consent was obtained from each patient.

\section{Histopathology and immunohistochemistry}

Paraffin-embedded sections were deparaffinized and rehydrated. For histopathology, the sections were stained with hematoxylin/eosin (HE). For immunohistochemistry, antigens were retrieved in citrate buffer, and the sections were blocked with $3 \%$ hydrogen peroxide and incubated with a primary antibody against p57 (1:100, sc56341, Santa Cruz Biotechnology, Santa Cruz, CA, USA) and anti-p-cofilin (Ser3) antibody (1:100, 11139, Signalway Antibody, Pearland, TX, USA) overnight at $4{ }^{\circ} \mathrm{C}$. Control sections were incubated with an isotype-matched control antibody. Next, the sections were incubated with biotinconjugated secondary antibodies for $30 \mathrm{~min}$ and streptavidin-peroxidase for $30 \mathrm{~min}$. Immunoreactive products were stained with 3,3'-diaminobenzidine and subsequently counterstained with hematoxylin. Finally, the sections were examined with a microscope (Q550CW; Leica, Manheim, Germany). For the evaluation of p57 and p-cofilin protein expression, the staining intensity was graded and scored as follows: 0 , no staining; 1 , weak staining; 2, moderate staining; and 3, strong staining. The extent of the staining was graded as $1(\leq 25 \%), 2$ (26$50 \%), 3(51-75 \%)$ and 4 ( $\geq 76 \%)$ based on the percentage of positively stained cells [12]. The number of positive cells was assessed by counting 10 random fields at $\times 400$ magnification. The final immunohistochemical staining score was obtained by multiplying the

Table 1 Relationship between the subcellular distribution of p57 protein and clinicopathologic factors in cancerous tissues of hepatocellular carcinoma patients

\begin{tabular}{|c|c|c|c|c|c|}
\hline Variable & $n$ & $\begin{array}{l}\text { Nuclear p57 } \\
\left(x^{\bigotimes} \pm s\right)\end{array}$ & $P$ & $\begin{array}{l}\text { Cytoplasmic p57 } \\
\left(\bar{x}^{\otimes} \pm s\right)\end{array}$ & $P$ \\
\hline Tumor size & & & $0.016^{*}$ & & 0.199 \\
\hline$<50 \mathrm{~mm}$ & 18 & $4.82 \pm 3.10$ & & $4.32 \pm 2.92$ & \\
\hline$\geq 50 \mathrm{~mm}$ & 27 & $2.88 \pm 2.65$ & & $3.18 \pm 3.33$ & \\
\hline Histological grade & & & 0.283 & & 0.503 \\
\hline $1+\|$ & 36 & $3.88 \pm 3.08$ & & $3.79 \pm 3.17$ & \\
\hline III & 9 & $2.83 \pm 2.48$ & & $3.08 \pm 3.37$ & \\
\hline TNM stage & & & 0.150 & & 0.098 \\
\hline $\mid+\|$ & 12 & $4.60 \pm 3.07$ & & $4.80 \pm 3.57$ & \\
\hline$I I I+I V$ & 33 & $3.30 \pm 2.89$ & & $3.20 \pm 2.97$ & \\
\hline \multicolumn{3}{|c|}{ Capsular invasion and extrahepatic metastasis } & 0.05 & & 0.013 \\
\hline Positive & 24 & $2.97 \pm 2.86$ & & $2.71 \pm 3.10$ & \\
\hline Negative & 21 & $4.54 \pm 2.93$ & & $4.83 \pm 2.96$ & \\
\hline AFP & & & 0.131 & & 0.670 \\
\hline$<400 \mu \mathrm{g} \cdot \mathrm{L}^{-1}$ & 21 & $4.32 \pm 2.84$ & & $3.84 \pm 3.13$ & \\
\hline$\geq 400 \mu \mathrm{g} \cdot \mathrm{L}^{-1}$ & 24 & $3.10 \pm 3.01$ & & $3.47 \pm 3.29$ & \\
\hline
\end{tabular}

$n$ Number of patients, AFP alpha-fetoprotein, TNM tumor-node-metastasis 
intensity and the extent of staining: A score of $0-2$ was considered to be negative expression; $3-5$, weak expression; 6-9, moderate expression; and 10-12, strong expression. A score of 6-12 was defined as positive staining, a score of $0-5$ was defined as markedly reduced expression or a lack of expression.

\section{Plasmids}

The p57 shRNA plasmids (pGPU6/GFP/Neo-shp57) were designed and synthesized by GenePharma Co. (Shanghai, China). The optimal p57 shRNAs had the following sequences: 5 '-CACCGCTTTAAGAGTCATTTATATT CAAGAGATATAAATGACTCTTAAAGCTTTTTTG3' (sense) and 5'-GATCCAAAAAAGCTTTAAGAGT CAT TTATATCTCTTGAATATAAATGACTCTTAAA GC-3' (antisense). The shRNA expression vector was pGPU6/GFP/Neo (GenePharma). The plasmid pGPU6/ GFP/Neo-shNC, which encodes a hairpin siRNA with a sequence that is not found in human genome databases, was used as a negative control.

\section{Cell culture, construction of stable transfectants and treatments}

The hepatoma cell lines HepG2, Hep3B, BEL7402, SMMC7721, and MHCC97H and the normal liver cell line L02 were obtained from Shanghai Cell Bank and cultured in DMEM (Invitrogen, Carlsbad, CA, USA) supplemented with $10 \%$ FBS (Invitrogen), penicillin $(100 \mathrm{IU} / \mathrm{ml})$ and streptomycin $(0.1 \mathrm{mg} / \mathrm{ml})$ at $37^{\circ} \mathrm{C}$ with $5 \% \mathrm{CO}_{2}$. BEL7402 cells and SMMC7721 cells were selected for our research. The cells were transfected with shp57 and shNC plasmids using Lipofectamine 2000 (Invitrogen) according to the manufacturer's protocols. After $24 \mathrm{~h}$, the cells were diluted and selected for 1 month using G418 (Invitrogen) at $600 \mu \mathrm{g} / \mathrm{ml}$ for BEL7402 cells and $800 \mu \mathrm{g} / \mathrm{ml}$ for SMMC7721 cells. Stable transfectants were confirmed by western blot and were maintained in medium containing G418 at $300 \mu \mathrm{g} / \mathrm{ml}$ for BEL7402 cells and $400 \mu \mathrm{g} / \mathrm{ml}$ for SMMC7721 cells. The stable p57 knockdown transfectants were named BEL7402-shp57 and SMMC7721-shp57. HCC cells transfected with shNC were named BEL7402-shNC and SMMC7721-shNC, which were used as controls.

\section{Reverse transcription PCR}

Total mRNA was extracted using TRIzol reagent (Invitrogen), and reverse transcription was performed using an RT-PCR kit (Takara, Dalian, China). cDNA synthesis was conducted with the SYBR ExScript RT-PCR kit (Takara) according to the manufacturer's instructions. The PCRs consisted of $5 \mathrm{~min}$ at $94{ }^{\circ} \mathrm{C}$ followed by 30 cycles of denaturation for $30 \mathrm{~s}$ at $94{ }^{\circ} \mathrm{C}$, annealing for $30 \mathrm{~s}$ at $58{ }^{\circ} \mathrm{C}$ and primer extension for $30 \mathrm{~s}$ at $72{ }^{\circ} \mathrm{C}$. The primer sequences for p57 were $5^{\prime}$-GCGGCGATCAAGAAGCTGT- $3^{\prime}$ and $5^{\prime}$-ATCGCCCGACGACTTCTCA-3'. The primer sequences for glyceraldehyde 3-phosphate dehydrogenase (GAPDH) were 5'-ACCACAGTCCATGCCATCAC-3' and 5'-TCCACCACCCTGTTGCTGTA-3'. Each measurement was performed in triplicate. GAPDH was applied as the internal housekeeping gene control.

\section{Western blot}

Cells were lysed using cell lysis buffer as previously reported [13]. Nuclear and cytoplasmic extracts were prepared as previously reported [14]. Equivalent amounts of protein were separated by SDS-PAGE (8-12\%) and transferred onto polyvinylidene fluoride membranes (Millipore, Danvers, MA, USA). The membranes were blocked and subsequently incubated with the following primary antibodies: anti-p57 antibody (1:500), anti-phosphocofilin (Ser3) antibody (1:300, 11139, Signalway Antibody, Pearland, TX, USA), anti- $\beta$-actin antibody (1:1000, sc-130301, Santa Cruz) and anti-lamin A antibody (1:600, 613501, Biolegend, San Diego, CA, USA). Blots were visualized with a secondary antibody conjugated to horseradish peroxidase (Santa Cruz) and an ECL detection system (Millipore). Western blotting was repeated three times for each protein.

\section{Co-immunoprecipitation and immunoblotting}

Cells were harvested and lysed in $0.1 \%$ NP-40 lysis buffer (50 mM Tris, pH 7.4, $250 \mathrm{mM} \mathrm{NaCl}, 5 \mathrm{mM}$ ethylenediaminetetraacetic acid, $50 \mathrm{mM} \mathrm{NaF}, 1 \mathrm{mM} \mathrm{Na} \mathrm{VO}_{4}, 0.1 \%$ NP-40, $0.02 \% \mathrm{NaN}_{3}, 1 \mathrm{mM}$ phenylmethylsulfonyl fluoride). For immunoprecipitation, $2 \mu \mathrm{g}$ of anti-p57 antibody was incubated with $50 \mu \mathrm{l}$ of Dyna-beads/protein G (Invitrogen) for $10 \mathrm{~min}$ at room temperature and subsequently incubated with $500-\mu \mathrm{l}$ cell lysate samples for $10 \mathrm{~min}$ at room temperature with gentle rotation to form Dynabead-Ab-Ag complexes. These complexes were then washed extensively with phosphate-buffered saline, resolved using $10 \%$ sodium dodecyl sulfate-polyacrylamide gel electrophoresis and immunoblotted with anti-LIMK1 antibody.

\section{Confocal immunofluorescence analysis}

Cells $\left(1 \times 10^{5}\right.$ per well $)$ were fixed with $4 \%$ paraformaldehyde and blocked with $5 \%$ bovine serum and $0.3 \%$ Triton X-100. The cells were then incubated with anti-p57 antibody (1:100) and anti-phospho-cofilin (Ser3) antibody (1:50) at $4{ }^{\circ} \mathrm{C}$ overnight followed by staining with TRITCconjugated anti-rabbit immunoglobulin (1:50, Santa Cruz) and $1 \mu \mathrm{g} / \mathrm{ml}$ DAPI (Roche). Immunofluorescence was visualized with a confocal laser scanning microscope (TCS SP5, Leica, Germany).

\section{Statistical analysis}

Descriptive data were assessed using Pearson's chisquare test (two-sided). Quantitative data were assessed 
by Student's $t$-test. $P<0.05$ was considered to be statistically significant. All statistical analyses were performed using SPSS 17.0 software for Windows (Chicago, IL).

\section{Results}

\section{Relationship between the subcellular localization of p57} protein and clinicopathologic factors of HCC

To determine the subcellular localization of p57 protein, immunohistochemistry was performed on 45 human HCC tissues and adjacent non-cancerous tissues. We noticed that p57 was expressed in both the nucleus and cytoplasm in the HCC tissues and the adjacent noncancerous liver tissues (Fig. 1a, b). In the HCC tissues, positive expression of $\mathrm{p} 57$ was detected in the nucleus in 14 samples and in the cytoplasm in 17 samples (Additional file 1: Figure S1). To determine the association between subcellular localization and p57 function, we further analyzed the relationship of the nuclear and cytoplasmic p57 with clinicopathological factors. The results showed that nuclear expression of $\mathrm{p} 57$ was reduced in specimens from patients with smaller tumors $(P<0.05)$ and that cytoplasmic expression of p57 was reduced in specimens from patients with capsular invasion and metastasis $(P<0.05)$ (Table 1$)$. These data indicated that the cytoplasmic localization of p57 may have a biological function related to $\mathrm{HCC}$ invasion.

\section{Association of cytoplasmic expression of p57 with p-cofilin in HCC tissues}

It is well known that LIMK1 phosphorylates cofilin at Ser- 3 and blocks the ability of cofilin to depolymerize actin filaments during cell migration [15]. Our previous data suggested that p57 downregulation accelerated the invasion of $\mathrm{HCC}$ cells by controlling the activity of LIMK1 and subsequently reducing the phosphorylation of cofilin [11]. To further elucidate whether p57 regulates HCC cell invasion in the cytoplasm, immunohistochemistry of p-cofilin was performed in human HCC tissues. The results indicated that p-cofilin was localized only in the cytoplasm (Fig. 1c). Moreover, we observed that of the samples with increased cytoplasmic p57 expression, 14 samples were positive for $\mathrm{p}$-cofilin expression, and of the samples with decreased cytoplasmic p57 expression, 15 samples were negative for $\mathrm{p}$-cofilin expression $(P<0.05)$. We further analyzed the association of cytoplasmic coexpression of p57 and p-cofilin with clinicopathological factors. The results showed that cytoplasmic coexpression of p57 and p-cofilin was reduced in specimens from patients with tumors at later stages (III + IV) and patients with capsular invasion and metastasis $(P<0.05)$ (Table 2). These data suggest that p57 and pcofilin might function in the cytoplasm to regulate HCC invasion.

\section{p57 regulates the level of p-cofilin in the cytoplasm of HCC cells}

We next investigated the role of $\mathrm{p}$-cofilin protein in p57-knockdown hepatoma cell lines. We selected the BEL7402 and SMMC7721 cell lines for the follow-up experiments because they exhibit moderate p57 expression, in contrast with the normal liver cell line LO2 (Fig 2a). Out of four p57 shRNAs, we selected p57shRNA4 because it induced optimal downregulation of p57 mRNA and protein (Fig 2b, c). Stable transfectants with downregulated p57 expression were constructed, and we observed that the level of p57 mRNA and protein was decreased in BEL7402-shp57 and SMMC7721-shp57 compared to BEL7402-shNC and SMMC7721-shNC (Fig 2d, e). Our previous experiments showed that p57 did not affect the level of LIMK1 but could affect the level of p-cofilin, which is the unique substrate of LIMK1 and reflects its activity [11]. However, the mRNA level of cofilin has no change in BEL7402-shp57 and SMMC7721-shp57 compared to BEL7402-shNC and SMMC7721-shNC cells (Additional file 2: Figure S2). We questioned whether p57 could directly bind LIMK1 to affect its activity. By coimmunoprecipitation, we further proved that p57 downregulation inhibited the assembly of p57 and LIMK1, indicating that p57 could combine with LIMK1 and regulate its kinase activity (Fig. 2f).
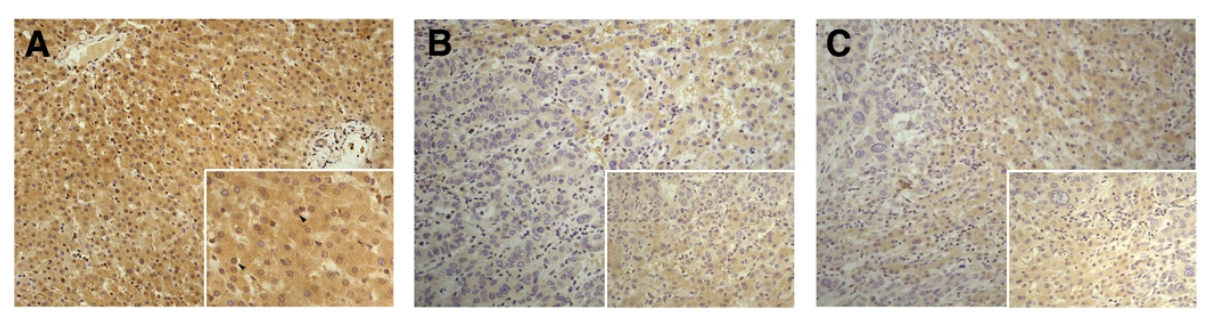

Fig. 1 Immunohistochemical staining of p57 and p-cofilin protein in cancerous tissues and adjacent non-cancerous tissues of hepatocellular carcinoma patients ( $\times 200)$. a Positive expression of p57 in both the nucleus and the cytoplasm of adjacent non-cancerous tissues. $\mathbf{b}$ In contrast with adjacent non-cancerous tissues, positive p57 expression in the cytoplasm is decreased in the specimen with extrahepatic metastasis. c Positive p-cofilin expression in the cytoplasm is decreased compared to adjacent non-cancerous tissues in the specimen with extrahepatic metastasis. Each inset shows images taken at $\times 400$ magnification 
Table 2 Association of cytoplasmic co-expression of p57 and p-cofilin with clinicopathologic factors in cancerous tissues of hepatocellular carcinoma patients

\begin{tabular}{|c|c|c|c|c|}
\hline \multirow[t]{2}{*}{ Variable } & \multirow[t]{2}{*}{$\mathrm{n}$} & \multicolumn{2}{|c|}{$\begin{array}{l}\text { Cytoplasmic co-expression of p57 and } \\
\text { p-cofilin }\end{array}$} & \multirow[t]{2}{*}{$P$} \\
\hline & & Positive & Non-positive & \\
\hline \multicolumn{4}{|l|}{ Tumor size } & 0.512 \\
\hline$<50 \mathrm{~mm}$ & 18 & 7 & 11 & \\
\hline$\geq 50 \mathrm{~mm}$ & 27 & 7 & 20 & \\
\hline \multicolumn{4}{|l|}{ Histological grade } & 0.428 \\
\hline $1+\|$ & 36 & 10 & 26 & \\
\hline III & 9 & 4 & 5 & \\
\hline \multicolumn{4}{|l|}{ TNM stage } & 0.004 \\
\hline $1+\|$ & 12 & 8 & 4 & \\
\hline III + IV & 33 & 6 & 27 & \\
\hline \multicolumn{4}{|c|}{ Capsular invasion and extrahepatic metastasis } & 0.009 \\
\hline Positive & 24 & 3 & 21 & \\
\hline Negative & 21 & 11 & 10 & \\
\hline \multicolumn{4}{|l|}{ AFP } & 0.520 \\
\hline$<400 \mu \mathrm{g} \cdot \mathrm{L}^{-1}$ & 21 & 8 & 13 & \\
\hline$\geq 400 \mu \mathrm{g} \cdot \mathrm{L}^{-1}$ & 24 & 6 & 18 & \\
\hline
\end{tabular}

$n$ Number of patients, AFP alpha-fetoprotein, TNM tumor-node-metastasis
To further verify whether p57 regulates the activity of LIMK1 in the cytoplasm, confocal immunofluorescence microscopy and western blot analysis of nuclear and cytoplasmic extracts were performed. In BEL7402-shNC and SMMC7721-shNC cells, we observed that p57 was expressed in both the nucleus and the cytoplasm, whereas p-cofilin was only expressed in the cytoplasm. p57 downregulation decreased the level of p-cofilin in the cytoplasm in BEL7402-shp57 and SMMC7721-shp57 cells (Fig. 3a, b). These findings demonstrated that p57 might affect the level of p-cofilin by regulating the kinase activity of LIMK1 in the cytoplasm.

\section{Discussion}

Increasing evidence has indicated that the Cip/Kip family of proteins has a role in cell migration and tumor metastasis [7]. Some clinical studies have shown that decreased expression of p57 is related to increased invasion and metastasis in cancers, categorizing this protein as a suppressor of tumor metastasis [16, 17]. Inducible expression of p57 in glioma cell lines deficient in this CKI reduces their motility and invasion [18]. In addition, p57 is involved in angiogenesis, which is an important event in cancer metastasis, via the regulation of vascular endothelial growth factor (VEGF) mRNA and protein levels [19]. p21 and p27, which are other Cip/Kip family members, were shown to regulate cell motility and invasion in the cytoplasm. However, the role of cytoplasmic p57 and its relationship with biological functions are unclear. Our study

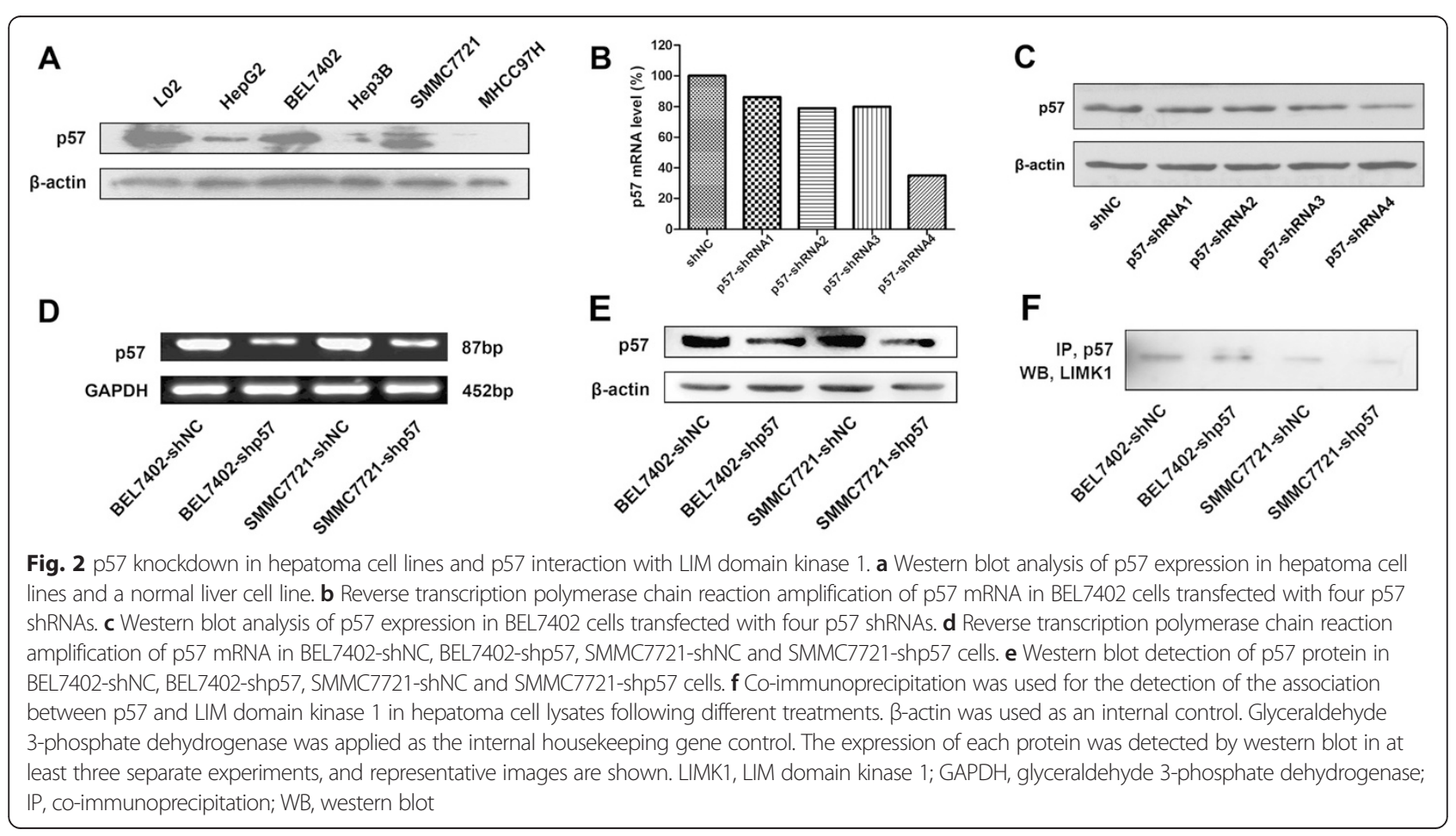


A

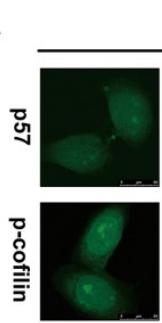

GFP
BEL7402-shNC
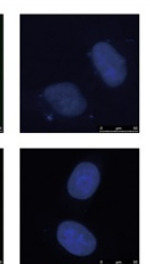

DAPI
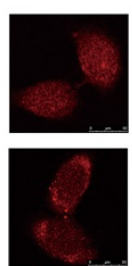

TRITC
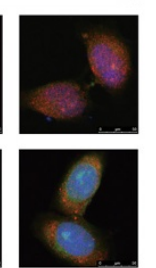

Merge

SMMC7721-shNC
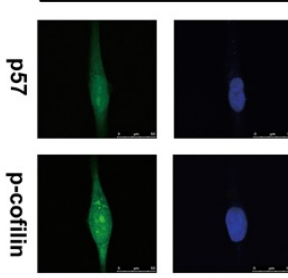

GFP

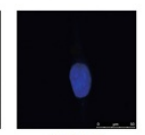

DAPI

B

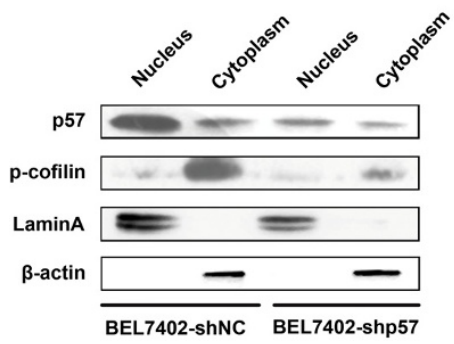

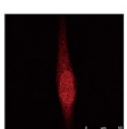

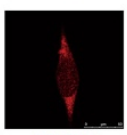

TRITC
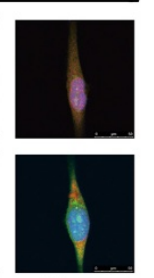

Merge

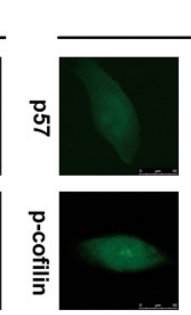

GFP

DAPI

SMMC7721-shp57
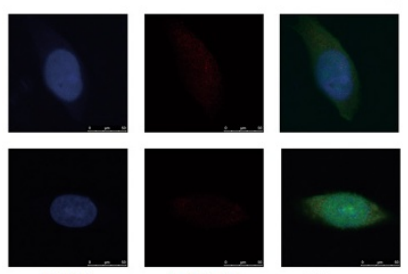

TRITC

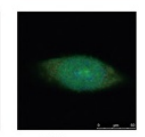

Merge

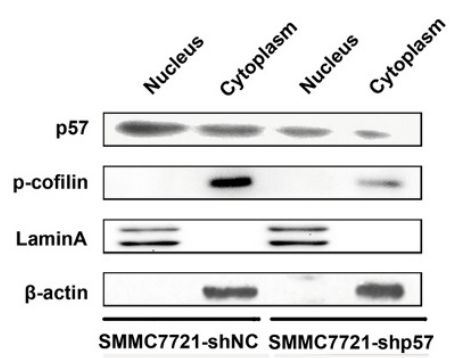

Fig. 3 p57 regulates the expression of p-cofilin in the cytoplasm. a Confocal immunofluorescence microscopy analysis of BEL7402-shNC, BEL7402-shp57, SMMC7721-shNC and SMMC7721-shp57 cells. Green fluorescence represents the transfected plasmid, red fluorescence shows the expression of the target protein, and blue fluorescence shows the nucleus. $\mathbf{b}$ Western blot analysis of nuclear and cytoplasmic extracts of p-cofilin in BEL7402-shNC, BEL7402-shp57, SMMC7721-shNC and SMMC7721-shp57 cells. $\beta$-actin was used as an internal control for cytoplasmic proteins. Lamin A was used as an internal control for nuclear proteins. GFP, Green fluorescent protein; TRITC, tetramethylrhodamine; DAPI, 4',6-diamidino-2-phenylindole

showed that p57 was localized to both the nucleus and the cytoplasm of normal liver cells and HCC cells. A correlation analysis indicated that nuclear p57 was reduced in specimens from patients with smaller tumors, and cytoplasmic p57 was reduced in specimens from patients with capsular invasion and metastasis. These results suggest that nuclear localization of p57 might regulate cellular proliferation and that cytoplasmic localization of p57 might regulate cell motility. However, the mechanism of action of p57 is different from that of p21 and p27. Cytoplasmic p21 was found to inhibit the activity of ROCK1, reduce the formation of actin stress fibers, and promote the invasion and metastasis of tumor cells [20]. In HCC, melanoma and breast cancer cells, cytoplasmic expression of p27 was found to promote migration and metastasis by inhibiting RhoA activity [21]. These findings indicate that cytoplasmic p21 and p27 might play a role as oncogenes in some human cancers. However, our results showed that cytoplasmic p57 inhibits the invasion and metastasis of HCC.
Cofilins are actin-binding proteins that play an essential role in regulating actin filament dynamics and reorganization by stimulating the severing and depolymerization of actin filaments in cell motility and cancer metastasis [22]. It is well known that cofilin is inactivated by phosphorylation by LIMK1, and the level of p-cofilin can reflect the activity of LIMK1. The interaction between p57 and LIMK1/p-cofilin has been reported in human cells [23, 24]. In HCC tissues, we found that cytoplasmic p57 was positively correlated with cytoplasmic p-cofilin. More importantly, coexpression of p57 and p-cofilin was reduced in specimens from patients with tumors at later stages (III + IV) and patients with capsular invasion and metastasis. Moreover, we found that p57 downregulation decreased the assembly of p57 and LIMK1. The results of confocal immunofluorescence microscopy and western blot analysis of nuclear and cytoplasmic extracts further supported the hypothesis that p57 could regulate the level of p-cofilin in the cytoplasm. These data suggested that p57 might regulate the kinase 
activity of LIMK1 and subsequently reduce the level of $\mathrm{p}$-cofilin in the cytoplasm to regulate $\mathrm{HCC}$ invasion.

Thus far, the mechanism regulating the subcellular localization of p57 has remained undefined, although it has been reported that phosphorylation and ubiquitination are two main mechanisms involved in this process. In breast cancer, Akt was found to interact with p57 and cause cytoplasmic localization of p57 by phosphorylating its Ser282 or Thr310 residue. Akt activity resulted in destabilization of $\mathrm{p} 57$ by accelerating the turnover rate of p57 and enhancing p57 ubiquitination. Importantly, the negative impact of HER2/Akt on p57 stability contributed to HER2-mediated cell proliferation, transformational activity and tumorigenicity [25]. These findings suggested that the AKT pathway might regulate p57 subcellular localization and subsequently affect its biological function. Importantly, phosphorylation is often coordinated with ubiquitination in the process of p57 degradation. In osteoblast cells, FBL12 was found to form an SCF (FBL12) complex and directly ubiquitinate p57, causing p57 degradation in a phosphorylation-dependent manner [26]. Another E3 ligase, Skp2, could also mediate the ubiquitination of p57 by collaborating with CSN6 in the cytoplasm to regulate tumor progression [27]. These studies indicated that p57 might be exported from the nucleus due to phosphorylation and degraded in the cytoplasm due to ubiquitination during tumor growth and invasion.

\section{Conclusions}

Our data indicated that reduced cytoplasmic p57 expression is associated with HCC invasion. Furthermore, p57 inhibits HCC invasion by regulating the level of p-cofilin in the cytoplasm via interaction with LIMK1, suggesting that cytoplasmic p57 may be a key regulator of HCC invasion. However, some questions remain concerning the mechanisms by which the subcellular localization of $\mathrm{p} 57$ regulates $\mathrm{HCC}$ progression. In the future, we will further investigate the mechanism of nuclear-cytoplasmic shuttling of p57. Additionally, we will explore components of this mechanism as promising targets for HCC prevention and therapy.

\section{Additional files}

Additional file 1: Figure S1. Immunohistochemical staining of nuclear p57 protein in adjacent non-cancerous tissues (left) and cancerous tissues (right) of hepatocellular carcinoma patients $(\times 200)$.

Additional file 2: Figure S2. p57 downregulation can not affects the mRNA level of p-cofilin in HCC cell lines. Reverse transcription PCR analysis of BEL7402-shNC, BEL7402-shp57, SMMC7721-shNC and SMMC7721-shp57 cells.

\section{Abbreviations}

CKI: Cyclin-dependent kinase inhibitor; RhoA: Ras homolog gene family, member A; ROCK1: Rho-associated, coiled-coil-containing protein kinase 1; LIMK1: LIM domain kinase 1; HCC: Hepatocellular carcinoma; VEGF: Vascular endothelial growth factor; UICC: International union against cancer; HE: Hematoxylin/eosin; GAPDH: Glyceraldehyde 3-phosphate dehydrogenase; AFP: Alpha-fetoprotein; TNM: Tumor-node-metastasis.

\section{Competing interests}

The authors declare that they have no competing interests.

\section{Authors' contributions}

HG acquired funding, designed the experiment, performed cell functional studies and drafted the manuscript. YL collected HCC tissues. TT carried out the molecular genetic studies. LH performed cell culture. ZR carried out the IHC experiments. XL performed the statistical analysis. WW acquired and analyzed data. KN performed general supervision of the research group. All authors read and approved the final manuscript.

\section{Acknowledgements}

This project was supported by the National Natural Science Foundation of China (NO. 81201923).

Received: 18 January 2015 Accepted: 16 July 2015

Published online: 15 August 2015

\section{References}

1. Matsuoka S, Edwards MC, Bai C, Parker S, Zhang P, Baldini A. p57KIP2, a structurally distinct member of the p21CIP1 Cdk inhibitor family, is a candidate tumor suppressor gene. Genes Dev. 1995;9:650-62.

2. Guo H, Tian T, Nan KJ, Wang WJ. p57: A multifunctional protein in cancer. Int J Oncol. 2010;36:1321-9.

3. Borriello A, Caldarelli I, Bencivenga D, Criscuolo M, Cucciolla V, Tramontano A, et al. p57(Kip2) and cancer: time for a critical appraisal. Mol Cancer Res. 2011;9:1269-84.

4. Kavanagh E, Joseph B. The hallmarks of CDKN1C (p57, KIP2) in cancer. Biochim Biophys Acta. 2011;1816:50-6.

5. Tesio M, Trumpp A. Breaking the cell cycle of HSCs by p57 and friends. Cell Stem Cell. 2011;9:187-92.

6. Besson A, Dowdy SF, Roberts JM. CDK inhibitors: cell cycle regulators and beyond. Dev Cell. 2008;14:159-69.

7. Besson A, Assoian RK, Roberts JM. Regulation of the cytoskeleton: an oncogenic function for CDK inhibitors? Nat Rev Cancer. 2004;4:948-55.

8. Vlachos P, Joseph B. The Cdk inhibitor p57(Kip2) controls LIM-kinase 1 activity and regulates actin cytoskeleton dynamics. Oncogene. 2009;28:4175-88.

9. Jemal A, Bray F, Center MM, Ferlay J, Ward E, Forman D. Global cancer statistics. CA Cancer J Clin. 2011;61:69-90.

10. Liu M, Jiang $L$, Guan XY. The genetic and epigenetic alterations in human hepatocellular carcinoma: a recent update. Protein Cell. 2014;5:673-91.

11. Guo H, Lv Y, Tian T, Hu TH, Wang WJ, Sui X, et al. Downregulation of p57 accelerates the growth and invasion of hepatocellular carcinoma. Carcinogenesis. 2011;32:1897-904.

12. Wong CC, Wong CM, Au SL, Ng IO. RhoGTPases and Rho-effectors in hepatocellular carcinoma metastasis: ROCK N'Rho move it. Liver Int. 2010;30:642-56.

13. Jin RJ, Lho Y, Wang Y, Ao M, Revelo MP, Hayward SW, et al. Down-regulation of p57Kip2 induces prostate cancer in the mouse. Cancer Res. 2008;68:3601-8.

14. Fan GK, Xu F, Yang B, Fujieda S. p57(kip2) expression is related to carcinogenesis and tumor progression in laryngeal tissues. Acta Otolaryngol. 2006;126:301-5.

15. Sakai K, Peraud A, Mainprize T, Nakayama J, Tsugu A, Hongo K, et al. Inducible expression of p57KIP2 inhibits glioma cell motility and invasion. J Neurooncol. 2004;68:217-23.

16. Matsuura T, Takahashi K, Nakayama K, Kobayashi T, Choi-Miura NH, Tomita $M$, et al. Increased expression of vascular endothelial growth factor in placentas of p57(Kip2) null embryos. FEBS Lett. 2002;532:283-8.

17. Lee S, Helfman DM. Cytoplasmic p21Cip1 is involved in Ras-induced inhibition of the ROCK/LIMK/cofilin pathway. J Biol Chem. 2004;279:1885-91.

18. Denicourt C, Saenz CC, Datnow B, Cui XS, Dowdy SF. Relocalized p27Kip1 tumor suppressor functions as a cytoplasmic metastatic oncogene in melanoma. Cancer Res. 2007;67:9238-43.

19. Mizuno K. Signaling mechanisms and functional roles of cofilin phosphorylation and dephosphorylation. Cell Signal. 2013;25:457-69. 
20. Tury A, Mairet-Coello G, Dicicco-Bloom E. The cyclin-dependent kinase inhibitor p57Kip2 regulates cell cycle exit, differentiation, and migration of embryonic cerebral cortical precursors. Cereb Cortex. 2011;21:1840-56.

21. Chow SE, Wan JS, Lin MR, Lee CL. Downregulation of p57kip2 promotes cell invasion via LIMK/cofilin pathway in human nasopharyngeal carcinoma cells. J Cell Biochem. 2011;112:3459-68.

22. Zhao R, Yang HY, Shin J, Phan L, Fang L, Che TF, et al. CDK inhibitor p57 (Kip2) is downregulated by Akt during HER2-mediated tumorigenicity. Cell Cycle. 2013;12:935-43.

23. Kim M, Nakamoto T, Nishimori S, Phan L, Fang L, Che TF, et al. A new ubiquitin ligase involved in p57KIP2 proteolysis regulates osteoblast cell differentiation. EMBO Rep. 2008;9:878-84.

24. Pateras IS, Apostolopoulou K, Koutsami M, Evangelou K, Tsantoulis P, Liloglou T, et al. Downregulation of the KIP family members p27(KIP1) and p57(KIP2) by SKP2 and the role of methylation in p57(KIP2) inactivation in nonsmall cell lung cancer. Int J Cancer. 2006;119:2546-56.

25. Matsumoto M, Furihata M, Ohtsuki Y, Sasaguri S, Ogoshi S. Immunohistochemical characterization of p57KIP2 expression in human esophageal squamous cell carcinoma. Anticancer Res. 2000;20:1947-52.

26. Tian T, Nan KJ, Wang SH, Liang X, Lu CX, Guo H, et al. PTEN regulates angiogenesis and VEGF expression through phosphatase-dependent and -independent mechanisms in HepG2 cells. Carcinogenesis. 2010;31:1211-9.

27. Joseph B, Wallen-Mackenzie A, Benoit G, Murata T, Joodmardi E, Okret S, et al. p57(Kip2) cooperates with Nurr1 in developing dopamine cells. Proc Natl Acad Sci U S A. 2003;100:15619-24.

\section{Submit your next manuscript to BioMed Central and take full advantage of:}

- Convenient online submission

- Thorough peer review

- No space constraints or color figure charges

- Immediate publication on acceptance

- Inclusion in PubMed, CAS, Scopus and Google Scholar

- Research which is freely available for redistribution 